\title{
BP-OP-3-7
}

\section{Survival improvement in locally advanced pancreatic cancer after FOLFIRINOX neoadjuvant treatment and conversion surgery}

Mirang LEE, Youngmin HAN, Jea Seung KANG, Hee Ju SOHN, Hyeong Seok KIM, Yoon Hyung KANG, Hongbeom KIM, Wooil KWON, Jin-Young JANG*

Surgery and Cancer Research Institute, Seoul National University College of Medicine, Seoul, Korea

Introduction: After the introduction of FOLFIRINOX, the treatment outcome has dramatically changed for advanced pancreatic cancers. However, aggressive surgical resection is still questionable due to the anatomical complexity with increased morbidity/mortality for locally advanced PDAC (LAPC). The aim of this study is to explore the possibility of conversion surgery and survival benefit in LAPC after FOLFIRINOX.

Methods: Of 349 patient with LAPC from 2011 to 2019, 279 patients who initially received FOLFIRINOX at least 4 cycles were enrolled. Patients were re-evaluated according to the NCCN guideline 2020, The responses were evaluated by RECIST criteria after FOLFIRINOX, and further treatment was decided in the multidisciplinary meeting. Surgical exploration was decided if eligibility criteria meet: (1) no deterioration of performance status with patient's willingness for surgery, (2) response to neoadjuvant treatment per RECIST criteria over SD with the possibility of R0 resection, (3) radiologic (including PET) no evidence of distant metastasis with nearly normalized serum CA19-9.

Results: Of 279 patients with LAPC, 47 (16.8\%) patients underwent curative resection. Resectability increased from 8\% (initial period) to $23 \%$ (late period). Partial response (PR) and stable disease (SD) were 35.8 and $47.3 \%$ after FOLFIRINOX. The median survival was significantly longer in the surgical group than in the chemotherapy group (56 m vs. $17 \mathrm{~m}, p<0.001)$. Regardless of response over SD, surgical resection group definitely showed markedly improved survival.

Conclusions: Surgical resection greatly affects survival improvement in LAPC with FOLFIRINOX. Aggressive resection should be considered if tumor responded to chemotherapy. 